\title{
CONTINUIDADE ESPACIAL PARA CARACTERÍSTICAS DENDROMÉTRICAS (NÚMERO DE FUSTES E VOLUME) EM PLANTIOS DE Eucalyptus grandis ${ }^{1}$
}

José Márcio de Mello², Frederico Silva Diniz ${ }^{3}$, Antônio Donizette de Oliveira², Carlos Rogério de Mello ${ }^{4}$, José Roberto Soares Scolforo ${ }^{2}$ e Fausto Weimar Acerbi Junior ${ }^{2}$

\begin{abstract}
RESUMO - O objetivo deste estudo foi verificar a continuidade espacial do número de fustes e do volume nas diferentes formas e intensidades amostrais de Eucalyptus grandis com idade entre 3 e 4 anos. A área de estudo abrangeu quatro talhões, totalizando 104,71 ha, pertencentes à Ripasa S/A Celulose e Papel. Os dados para a realização do estudo de variabilidade espacial foram coletados em parcelas circulares e em parcelas em linhas distribuídas sistematicamente na área, nas intensidades de 1:4 (1 parcela a cada 4 ha), 1:7 e 1:10. Foi possível verificar que, tanto em número de fustes quanto em volume, os dados apresentaram distribuição aproximadamente normal. Pela análise variográfica, foi verificado que as características número de fustes e volume de madeira apresentaram-se estruturadas espacialmente. O modelo exponencial foi o que se ajustou melhor aos semivariogramas experimentais das características nas diferentes formas de parcela e intensidade amostral. A continuidade espacial foi detectada em todas as intensidades amostrais e formas de parcelas avaliadas, quanto a número de fustes. Portanto, o uso da estatística espacial no processamento dessa variável aumentará a precisão das estimativas. No caso de volume, na intensidade amostral 1:10 não foi possível detectar continuidade espacial. Em tal condição, deve-se utilizar a estatística clássica para processamento do inventário florestal.
\end{abstract}

Palavras-chaves: Biometria florestal, inventário florestal e manejo florestal.

\section{SPATIAL CONTINUITY FOR DENDROMETRIC CHARACTERISTICS (TRUNK NUMBER AND WOOD VOLUME) IN Eucalyptus grandis PLANTATIONS}

\begin{abstract}
This study aimed to verify the spatial continuity of trunk number and wood volume in different plot shapes and sample intensities of Eucalyptus grandis plantations with age varying from three to four years. The study area includes four stands, totalizing 104.71 hectares owned by Ripasa S/A Cellulose and Paper Company. The data for accomplishing the spatial variability study were collected in circular and line plots distributed systematically in the area at 1:4, 1:7 and 1:10 intensities. It was possible to verify that both for trunk number and volume, the data have presented, approximately, normal distribution. Based on variogram analysis, trunk number and wood volume variables were found to be spatially structured. The exponential semi-variogram model was the best model fitted to the experimental semi-variograms of the variables in different plot shapes and sample intensities. The spatial continuity of the trunk number variable was detected at all sample intensities and plot shapes. Therefore, the use of spatial statistics to process this variable will increase the precision of the estimates. Considering the volume, at sample intensity 1:10, we could not evaluate the existence of a spatial continuity structure. Under this condition, classic statistics should be used to process the forest inventory.
\end{abstract}

Keywords: Forest biometry, forest inventory and forest management.

\footnotetext{
${ }^{1}$ Recebido em 16.08.2007 e aceito para publicação em 26.01.2009.

${ }^{2}$ Departamento de Ciências Florestais da Universidade Federal de Lavras (UFLA). E-mail: <josemarcio@ufla.br>; < donizete@ufla.br>; <jscolforo@ufla.br>;<fausto@ufla.br>.

${ }_{3}^{3}$ Ripasa S/A Celulose e Papel. E-mail: <dfrederico@am.ripasa.com.br>.

${ }^{4}$ Departamento de Engenharia da UFLA E-mail: <crmello@ufla.br>.
} 


\section{INTRODUÇÃO}

Em florestas plantadas, há grande variação no espaçamento de plantio, a qual pode ser ocasionada por fatores como: erros cometidos no processo de plantio, ocorrência de situações topográficas que impedem a realização dessa operação de forma correta e mortalidade natural que existe na floresta, entre outras. Todas essas questões causam impacto na estimativa do número de plantas e, consequentemente, no volume de madeira. Uma das maneiras de se conhecerem as variações no número de plantas de um povoamento florestal é por meio do inventário. A capacidade da amostra retirada numa floresta em estimar as características desejadas está relacionada à forma e, principalmente, à intensidade amostral (SCOLFORO e MELLO, 2006).

Os métodos estatísticos tradicionais aplicados ao inventário florestal utilizam uma medida central (média) e uma medida de dispersão (variância) para descrever determinada variável dendrométrica. Contudo, não levam em consideração as possíveis correlações entre observações vizinhas e, portanto, não exploram suficientemente as relações que possam existir entre as unidades amostrais (MELLO et al., 2005). Para suprir essa deficiência, a geoestatística, por ser um conjunto de técnicas que descreve a autocorrelação espacial entre as unidades amostrais, possibilitando a geração de mapas com diferentes classes de produtividade sem viés estatístico, surge como alternativa viável para aumentar a precisão nos inventários florestais (MELLO, 2004).

A possibilidade do uso de técnicas geoestatísticas, no intuito de aprimorar o processamento dos inventários florestais, é uma ação que deve ser avaliada constantemente, a fim de verificar se há ou não relação entre as unidades amostrais. A avaliação da estrutura de continuidade espacial de dada característica é realizada com base na Teoria de Variáveis Regionalizadas, que foi descrita por Matheron (1963) e Journel e Huijbregts (1978). Os primeiros relatos a respeito da continuidade espacial de informações florestais foram descritos por Matérn (1960) e Osborne (1942). Esses autores trabalharam com volumes de madeira oriunda de parcelas distribuídas sistematicamente na área. $\mathrm{Na}$ área de tecnologia da madeira já existem estudos avaliando o potencial da geoestatística aplicado à dureza da madeira (LIMA et al., 2006).

Segundo Mello (2004), na presença de correlação espacial das características dendrométricas há redundância de informação quando se utilizam os procedimentos "clássicos" de amostragem. Neste trabalho, em razão da correlação espacial da característica volume e do número total de parcelas lançadas na área, parte delas não carregava informação sobre a variabilidade espacial do volume. Essa fração de parcelas provocou redundância de informação, gerando uma superestimativa da precisão do inventário. Com o uso da geoestatística, esse problema poderá ser minimizado ou, mesmo, corrigido, modelando parcela do erro aleatório associado à dependência espacial.

Este trabalho teve como objetivo verificar a continuidade espacial do número de fustes e do volume, nas diferentes formas e intensidades amostrais, em Eucalyptus grandis, com idade variando de 3 a 4 anos.

\section{MATERIAL E MÉTODOS}

\subsection{Caracterização da área e coleta dos dados}

A área de estudo abrangeu quatro unidades de manejo (talhões) de Eucalyptus grandis, totalizando 104,71 ha, com idades variando de 3 a 4 anos, de primeira rotação. A área pertence à empresa Ripasa S/A Celulose e Papel e o plantio localizavam-se no Município de Avaré, SP.

Os dados para a realização do estudo de continuidade espacial foram coletados em parcelas circulares e em parcelas em linhas, distribuídas sistematicamente na área. A justificativa para usar a parcela em linha, em plantios de eucalipto, é que as unidades no formato tradicional (circular ou retangular) não captam suficientemente bem o número de fustes por hectare. Isso pode ocorrer devido a diversos fatores, destacando-se erros de espaçamento e erros internos no mapeamento. No segundo caso, trata-se de situações que não são delimitadas no mapa, como estradas e carreadores abandonados, afloramento de rochas, manchas de florestas nativas e bacias de contenção de água, entre outros. A hipótese foi de que a parcela em linha consegue captar esses problemas ocorridos no mapeamento e no espaçamento de plantio.

O ponto de referência para delimitar a parcela em linha foi o centro da diagonal entre duas árvores opostas de linhas adjacentes da parcela circular (Figura 1).

A partir do ponto central da parcela circular, definiuse a parcela em linha de $120 \mathrm{~m}$ de comprimento, obedecendo ao alinhamento de plantio, no qual se contou 
o número de covas e de fustes. Do ponto central, caminharam-se $60 \mathrm{~m}$ para uma direção e $60 \mathrm{~m}$ para a direção oposta. A partir do ponto central, traçou-se uma linha de $50 \mathrm{~m}$, perpendicular à linha de plantio. Assim, pôde-se determinar o espaçamento médio de plantio. As intensidades amostrais das parcelas em linha foram idênticas às das parcelas circulares, ou seja, de 1:4 (1 parcela a cada 4 ha); $1: 7$ e 1:10.

Para se obter o espaçamento médio de plantio, contou-se o número de covas (árvores vivas e falhas) nas duas linhas centrais, ao longo dos $120 \mathrm{~m}$. A razão entre o comprimento $(120 \mathrm{~m})$ e o número de covas forneceu o espaçamento médio na linha. $\mathrm{Na}$ linha de $50 \mathrm{~m}$, perpendicular ao maior sentido, contou-se o número de linhas de plantio. Mediu-se, também, a distância entre as duas linhas centrais. A razão do comprimento da linha $(50 \mathrm{~m})$ pelo número de linhas possibilitou gerar o espaçamento médio entre linhas de plantio. Cada parcela em linha, dentro do talhão, gerou um valor de espaçamento médio. Por meio do volume médio por árvore, gerado pelo inventário florestal contínuo, juntamente com o número de fustes/ha da parcela em linha, obteve-se o volume médio do talhão. Dessa forma, foi possível definir o número de fustes e o volume de cada talhão avaliado.

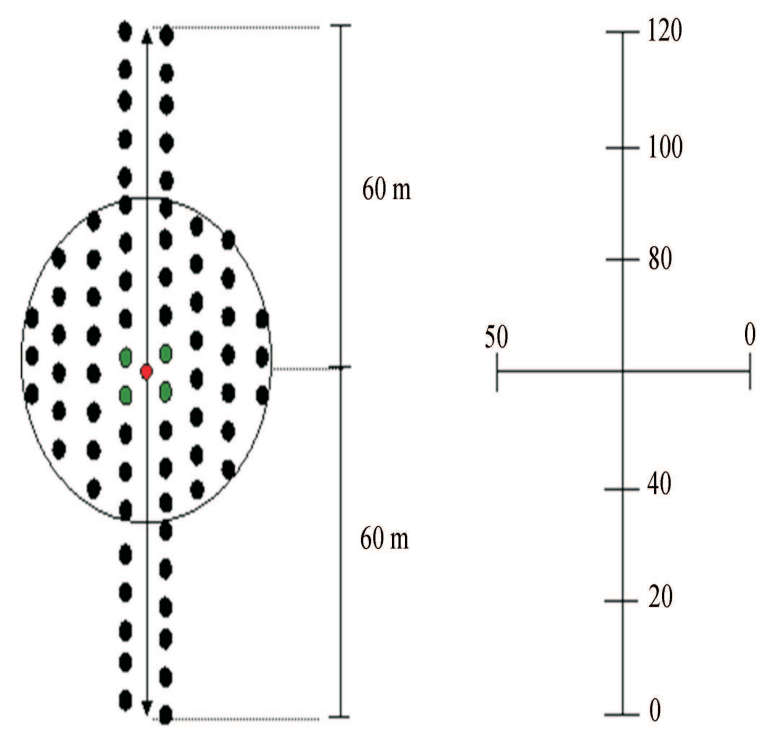

Figura 1 - Esquema ilustrativo do lançamento de uma parcela em linha.

Figure 1 - Illustrative scheme of the design of the in line plot design.

\section{Estudo variográfico}

A primeira etapa de uma análise geoestatística consiste do estudo exploratório dos dados. Por esse estudo, foi possível verificar a tendência das características avaliadas em função da longitude (WE) e da latitude (NS). Além disso, foi possível observar o comportamento dos dados relacionados à forma, à distribuição e à tendência central (Normalidade). Embora essa análise não considere a estrutura de correlação espacial, ela permitiu identificar a presença de dados discrepantes que, segundo Cressie (1993), afeta fortemente a verificação da continuidade espacial.

As características número de fustes e volume foram consideradas uma variável regionalizada $\mathrm{Z}$, que pode variar continuamente num espaço geográfico. Cada parcela, seja circular ou em linha, foi georreferenciada. Portanto, cada valor observado $z$ (xi), nos locais $x i, i=1,2, \ldots n$, em que xi é a coordenada geográfica da parcela, é considerada uma realização da variável aleatória $\mathrm{Z}(\mathrm{x})$.

O estudo da correlação entre as variáveis mensuradas, em cada ponto z (xi), é efetuado por meio do semivariograma. O semivariograma é o instrumento geoestatístico que avalia a variância entre pares de pontos, separados por uma distância $h$. Na ausência de correlação entre as observações efetuadas em diferentes pontos, a variância da variável regionalizada não é afetada pela distância que separa os pontos observados. Trata-se de uma situação na qual os dados são considerados independentes. Com os dados levantados em campo, foram construídos os semivariogramas experimentais, os quais foram modelados por meio de ajuste de modelos teóricos de semivariogramas, avaliando-se a qualidade dos respectivos ajustes. Esse ajuste é conduzido estimando-se os parâmetros do semivariograma, os quais, resumidamente, são: efeitopepita, que diz respeito ao erro aleatório não captado pela amostragem (erro na pequena escala); patamar, que descreve a estrutura de dependência espacial; e alcance, significando a distância na qual a dependência espacial existe e deve ser considerada.

Ajustou-se, dessa forma, o modelo exponencial, apresentado por Journel e Huijbregts (1978) e Mc Bratney e Webster (1986), das características número de fustes e volume, a fim de se obter o conjunto de parâmetros para as respectivas funções. Os ajustes foram feitos pelo método da máxima verossimilhança (DIGGLE e RIBEIRO JR., 2007).

R. Árvore, Viçosa-MG, v.33, n.1, p.185-194, 2009 
A avaliação do grau de dependência espacial (GDE) das características foi realizada a partir do porcentual de variação estruturada $\left(\sigma^{2}\right)$ em relação ao patamar $\left(\tau^{2}+\sigma^{2}\right)$, apresentado por Biondi et al. (1994). Essa mesma relação foi utilizada em trabalhos desenvolvidos por Novaes Filho et al. (2007). Essa medida foi útil para comparar a estrutura de continuidade espacial da característica número de fustes e volume nos diferentes talhões.

A comparação do grau de continuidade espacial entre as formas de parcelas e as diferentes intensidades amostrais foi efetuada a partir dos gráficos escalonados dos respectivos semivariogramas, auxiliados com o grau de dependência espacial (MELLO et al., 2005).

Além da comparação visual dos semivariogramas escalonados, foram efetuados mapas de krigagem, a fim de comparar o modelo espacial em diferentes intensidades amostrais e entre formas diferentes de parcelas. Tal comparação se deu por meio do porcentual de diferença de área em cada classe de produtividade do mapa de krigagem. A comparação foi realizada apenas com relação à variável volume $\left(\mathrm{m}^{3} / \mathrm{ha}\right)$.
Todas as análises geoestatísticas foram desenvolvidas com o programa $\mathrm{R}$, no pacote geoR (RIBEIRO JÚNIOR e DIGGLE, 2001).

\section{RESULTADOS E DISCUSSÃO}

\subsection{Análise exploratória dos dados}

Na Figura 2ab, apresentam-se os gráficos que caracterizam o comportamento da variável número de fustes em relação às coordenadas geográficas (longitude e latitude, respectivamente), para a intensidade amostral 1:4, no caso de parcela de forma circular. Na Figura $2 \mathrm{~cd}$, destaca-se essa mesma relação para a variável volume por hectare. Nas intensidades 1:7 e 1:10, nas formas de parcela circular e em linha, o comportamento dessa relação foi semelhante. Verificou-se que houve tendência entre as variáveis, com relação ao sentido leste-oeste, mas não no norte-sul. Mediante tendência detectada, esta foi removida, para a análise variográfica. A condição de não-tendência é fundamental para se admitir a Hipótese Intrínseca, descrita por Isaaks e Srivastava (1989), Journel e Huijbrets (1978), Ribeiro Júnior (1995) e Vieira (2000).

a)
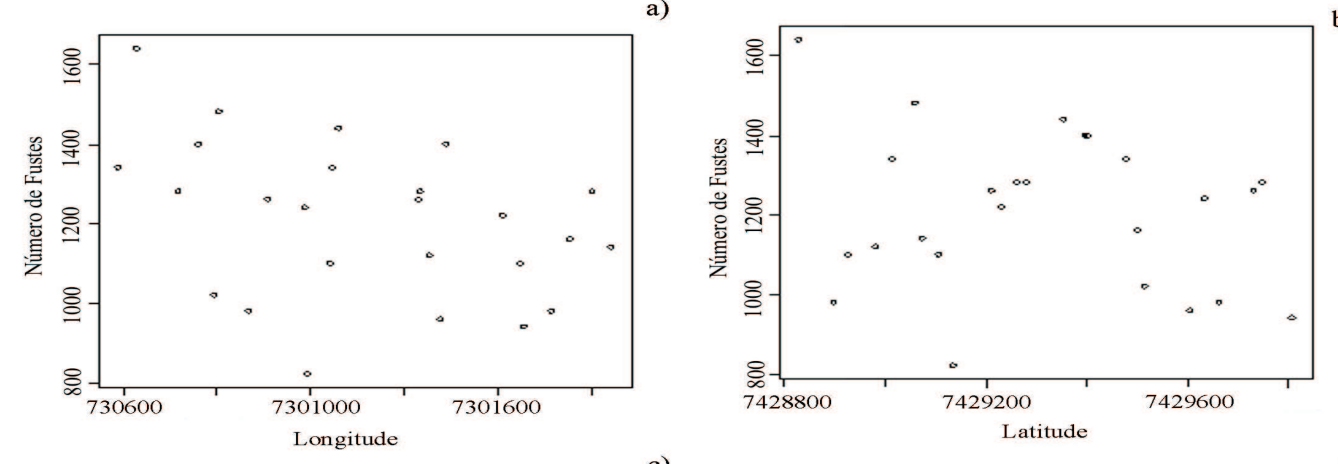

b) c)
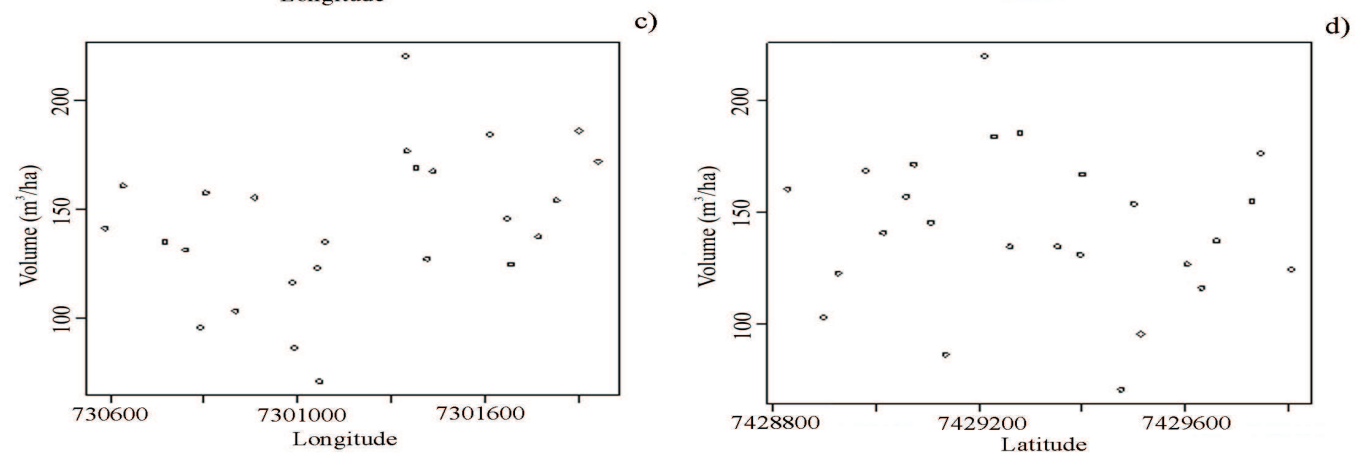

Figura 2 - Relação entre número de fustes $(a, b)$ e volume (c,d) com as coordenadas geográficas longitude e latitude. Figure 2 -Relationship between number of trunks $(a, b)$ and volume $(c, d)$ with the geographic coordinates longitude and latitude.

R. Árvore, Viçosa-MG, v.33, n.1, p.185-194, 2009 
Na Figura 3, apresentam-se os gráficos de normalidade do número de fustes e volume. Notase, nessa figura, que os dados apresentaram distribuição aproximadamente normal de ambas as variáveis. Essa é uma condição plausível para se utilizar o método de ajuste do semivariograma da máxima verossimilhança (DIGGLE e RIBEIRO JÚNIOR, 2007). O teste de Shapiro-Wilks não foi significativo para as duas características avaliadas, aceitandose, portanto, que os dados possuíam distribuição aproximadamente normal.

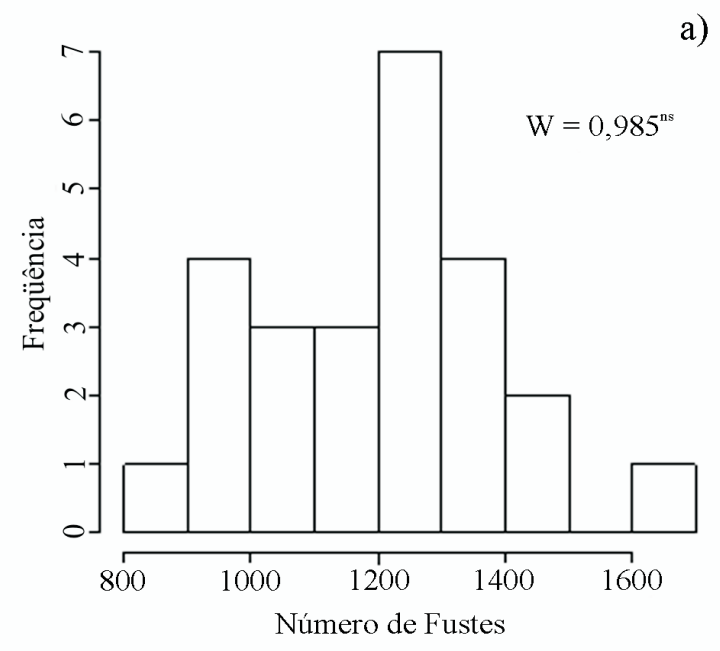

a)
Na Figura 4 estão os gráficos box plot das duas características avaliadas. Eles são úteis na análise exploratória, pois permitem identificar dados discrepantes ou "outliers", que possuem comportamento diferente do apresentado pela maioria das informações (BUSSAB e MORETTIN, 2002). Os dados discrepantes podem distorcer a variância do ruído (efeito-pepita), provocando falsa existência da correlação espacial (DIGGLE e RIBEIRO JÚNIOR, 2007). Pela análise desses dados, não foi detectado nenhum "outlier”, não havendo informação significativamente diferente das demais.

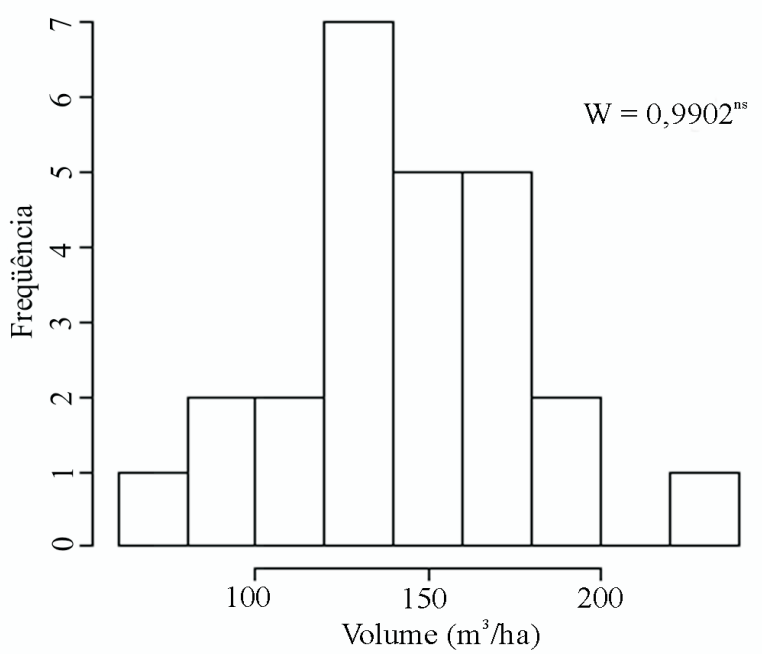

b)

Figura 3 - Gráficos de normalidade do número de fustes (a) e volume (b) por hectare.

Figure 3 - Normality graphs for number of trunks $(a)$ and volume $(b)$ per hectare.

a)
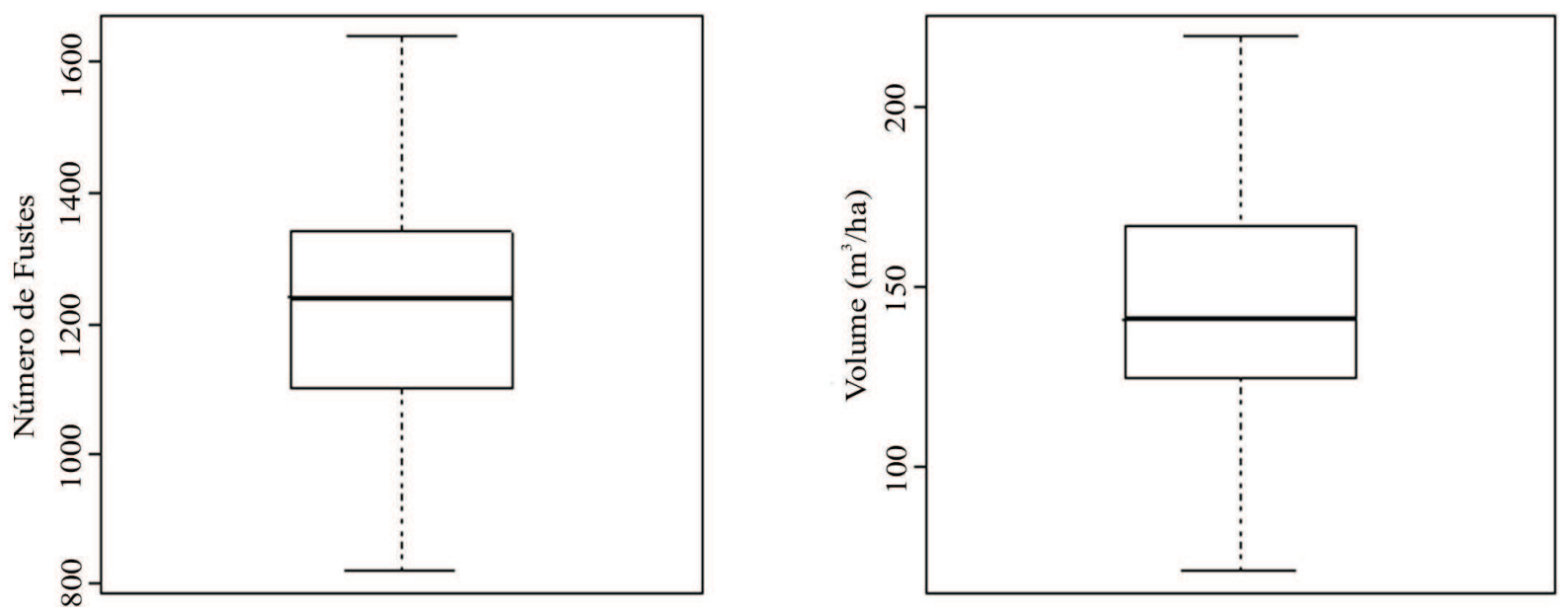

Figura 4 - Gráficos de box plot do número de fustes (a) e volume por hectare (b).

Figure 4-Box plot graphs for number of trunks $(a)$ and volume $(b)$ per hectare. 


\subsection{Análise da estrutura de continuidade espacial}

Pela análise variográfica, foi possível detectar que as duas características avaliadas apresentaram-se estruturadas espacialmente. Portanto, há um modelo espacial que modela essa estrutura. O modelo exponencial foi o que se ajustou melhor aos semivariogramas experimentais das características nas diferentes formas de parcela e intensidade amostral. $\mathrm{Na}$ intensidade amostral de uma parcela a cada 10 ha, a característica volume ( $\mathrm{m}^{3} / \mathrm{ha}$ ) não apresentou estrutura modelável para as duas formas de parcelas. Os parâmetros ajustados do modelo exponencial, nas diferentes situações avaliadas, estão apresentados na Tabela 1 .

Pelo grau de dependência espacial (GDE), a variável número de fustes apresentou-se fortemente estruturada (100\%), para os dois tipos de parcelas, nas três intensidades avaliadas, exceto na intensidade $1: 4$. No caso de volume, exceto na intensidade de 1:10, também houve forte estrutura de continuidade espacial, conforme classificação de Cambardella et al. (1994). Resultado semelhante para volume também foi obtido por Mello et al. (2005), trabalhando com povoamentos de eucalipto.

Os resultados indicaram que, nas estimativas volumétricas em povoamentos de eucalipto, na intensidade amostral de no máximo 1 parcela a cada 7 ha (1:7), deve-se utilizar a componente espacial, a fim de obter estimativas fidedignas da precisão do inventário. Conforme pode ser observado nos trabalhos de Cochran (1977) e Thompson (1992), desconsiderando a correlação espacial, há superestimativa da precisão, conduzindo a erros de planejamento, com possíveis aumentos nos custos do inventário.

Para a intensidade amostral de uma parcela a cada 10 ha, a estrutura espacial não contribuirá para a otimização do processamento do inventário florestal, ou seja, redução da variância por meio da correlação entre amostras. Nessa escala de observação, as informações devem ser tratadas de forma independente, com aplicação da estatística clássica.

Os valores do alcance $(\phi)$, apresentados na Tabela 1 , referem-se ao alcance do modelo exponencial, conforme descrito por Journel e Huijbregts (1978) e por McBratney e Webster (1986). Independentemente da forma da parcela e da intensidade amostral, o alcance médio do número de fustes e de volume foi de aproximadamente $270 \mathrm{~m}$ e de $280 \mathrm{~m}$, respectivamente. Esses valores podem ser observados nos semivariogramas escalonados das Figuras 5 e 6 . Com relação ao volume $\left(\mathrm{m}^{3} / \mathrm{ha}\right)$, Mello et al. (2005), trabalhando com florestas de eucalipto na Região Sul do Estado de São Paulo, encontraram valor do alcance de $250 \mathrm{~m}$. Assim, em planos amostrais com distâncias entre parcelas acima de $280 \mathrm{~m}$, as unidades amostrais devem ser consideradas independentes. Caso contrário, qualquer método estatístico empregado para análise deve considerar a componente espacial, evitandose, assim, problemas associados à superestimativa do volume existente no povoamento.

Na Figura 5, compara-se, com base no semivariograma escalonado, o comportamento da estrutura espacial da variável número de fustes das parcelas circulares e das parcelas em linha. Em cada forma de parcela, a estrutura espacial foi detectada nas três intensidades avaliadas. Apesar de os parâmetros dos modelos serem diferentes, a forma da estrutura foi semelhante nas intensidades estudadas. Portanto, em número de fustes a intensidade amostral não proporcionou forte impacto sobre a estrutura espacial. Os semivariogramas que caracterizaram a estrutura de continuidade espacial para volume, nas intensidades de 1:4 e 1:7, foram semelhantes, conforme pode ser avaliado na Figura 6, uma vez que, na forma escalonada, não se observaram diferenças significativas entre os semivariogramas.

A estrutura de continuidade espacial foi semelhante, em cada intensidade amostral, para número de fustes entre a parcela circular e a parcela em linha. Esse mesmo comportamento foi verificado na variável volume (Figura 7). Ao comparar a forma e intensidade amostrais, observou-se que essas fontes de variação influenciaram pouco a detecção da estrutura espacial, exceto o volume, com a intensidade de 1:10. Assim, estudos com parcelas circulares ou em linha numa intensidade intermediária àquela dos inventários pré-corte e contínuo podem motivar futuros trabalhos. A intensidade de 1:7 pode atender às necessidades do inventário pré-corte e contínuo. Usando os benefícios da correlação espacial entre as parcelas, pode-se aumentar a precisão das estimativas dos inventários florestais. Com essa intensidade amostral é possível atender a duas demandas importantes do planejamento florestal da empresa, que são a precisão das informações com relação ao crescimento da floresta (monitoramento) e o fornecimento de informações imediatamente antes do corte. Essas informações são úteis para o planejamento da colheita florestal. 
Tabela 1 - Parâmetros do modelo exponencial do efeito-pepita $\left(\tau^{2}\right)$, variação estruturada $\left(\sigma^{2}\right)$, patamar $\left(\tau^{2}+\sigma^{2}\right)$, alcance $(\phi)$ e o grau de dependência espacial (GDE\%), em número de fustes e volume, nas diferentes formas e intensidades amostrais

Table 1 - Parameters from the nugget exponential model $\left(\tau^{2}\right)$, sill $\left(\sigma^{2}\right)$, sill total $\left(\tau^{2}+\sigma^{2}\right)$, range $(\phi)$, and spatial dependence degree (GDE\%), for number of trunks and volume, in different plot shapes and sample intensities

\begin{tabular}{|c|c|c|c|c|c|c|c|c|c|c|}
\hline \multirow{2}{*}{$\begin{array}{l}\text { Parcela- } \\
\text { Intensidade }\end{array}$} & \multicolumn{5}{|c|}{ Número de Fustes } & \multicolumn{5}{|c|}{ Volume $\left(\mathrm{m}^{3} / \mathrm{ha}\right)$} \\
\hline & $\tau^{2}$ & $\sigma^{2}$ & $\tau^{2}+\sigma^{2}$ & $\phi(\mathrm{m})$ & $\operatorname{GDE}(\%)$ & $\tau^{2}$ & $\sigma^{2}$ & $\tau^{2}+\sigma^{2}$ & $\phi(\mathrm{m})$ & GDE(\%) \\
\hline Circular-1:4 & 0 & $36.276,8$ & $36.276,8$ & 55 & 100 & 0 & $1.089,2$ & $1.089,2$ & 117 & 100 \\
\hline Circular-1:7 & 0 & $27.002,2$ & $27.002,2$ & 125 & 100 & 0 & $1.142,2$ & $1.142,2$ & 85 & 100 \\
\hline Circular-1:10 & 0 & $28.609,5$ & $28.609,5$ & 73 & 100 & $1.226,8$ & 0,0 & $1.226,8$ & 0 & 0 \\
\hline Linha-1:4 & 10 & $17.376,8$ & $27.376,8$ & 56 & 63,5 & 0 & 986,5 & 986,5 & 121 & 100 \\
\hline Linha-1:7 & 0 & $23.380,9$ & $23.380,9$ & 92 & 100 & 0 & $1.121,4$ & $1.121,4$ & 59 & 100 \\
\hline Linha-1:10 & 0 & $29.437,7$ & $29.437,7$ & 140 & 100 & $1.240,2$ & 0,0 & $1.240,2$ & 0 & 0 \\
\hline
\end{tabular}

a)
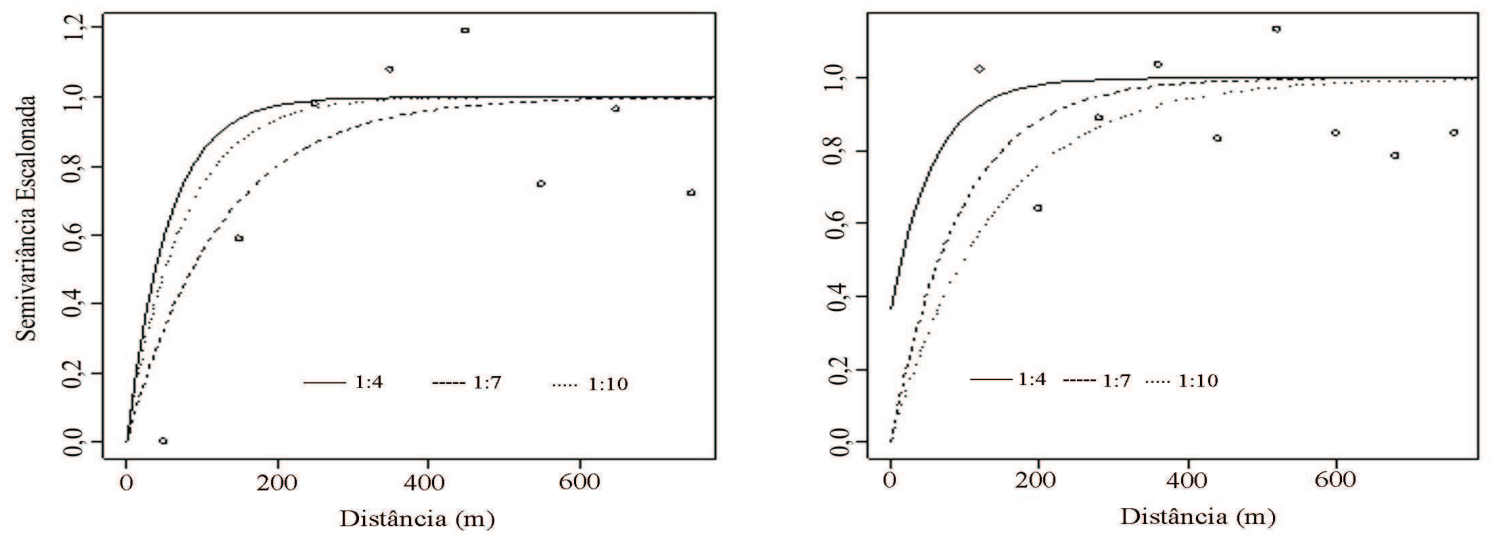

Figura 5 - Semivariogramas escalonados comparando intensidade amostral do número de fustes em parcelas circulares (a) e parcelas em linha (b).

Figure 5 - Scaled semivariograms comparing sample intensity for number of trunks in circular sample plots (a) and in line sample plots $(b)$.

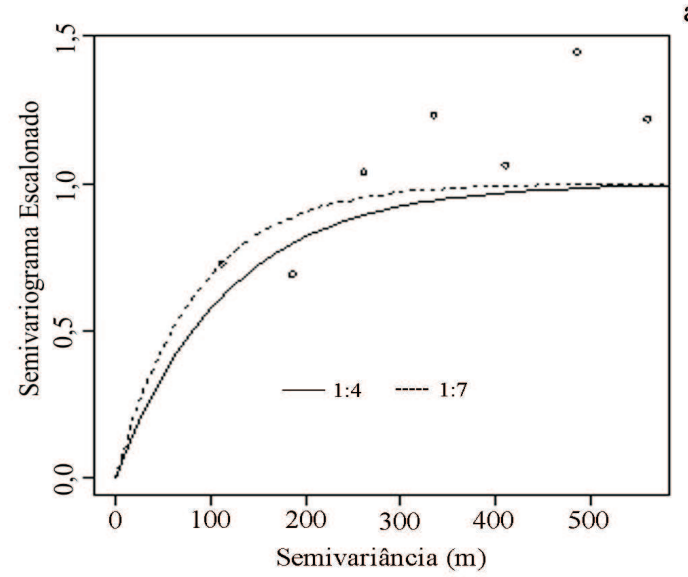

a)

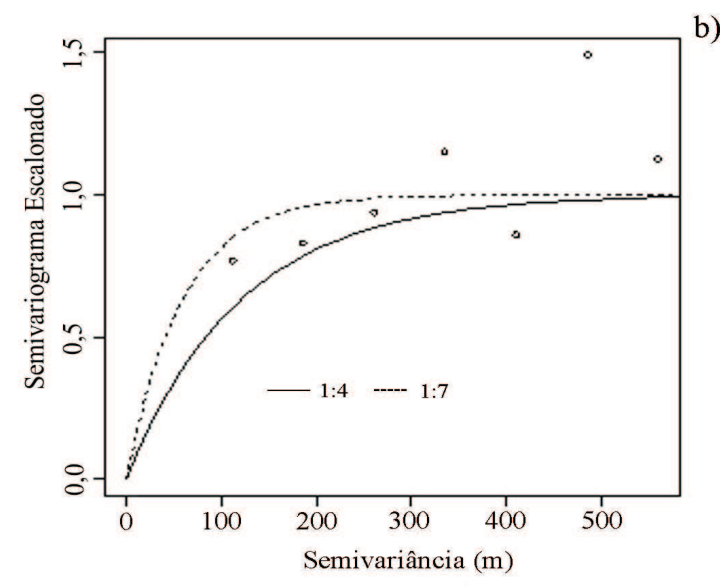

Figura 6 - Semivariogramas escalonados comparando intensidade amostral do volume em parcelas circulares (a) e parcelas em linha (b).

Figure 6 - Scaled semivariograms comparing sample intensity for volume in circular sample plots (a) and in line sample plots (b). 
a)
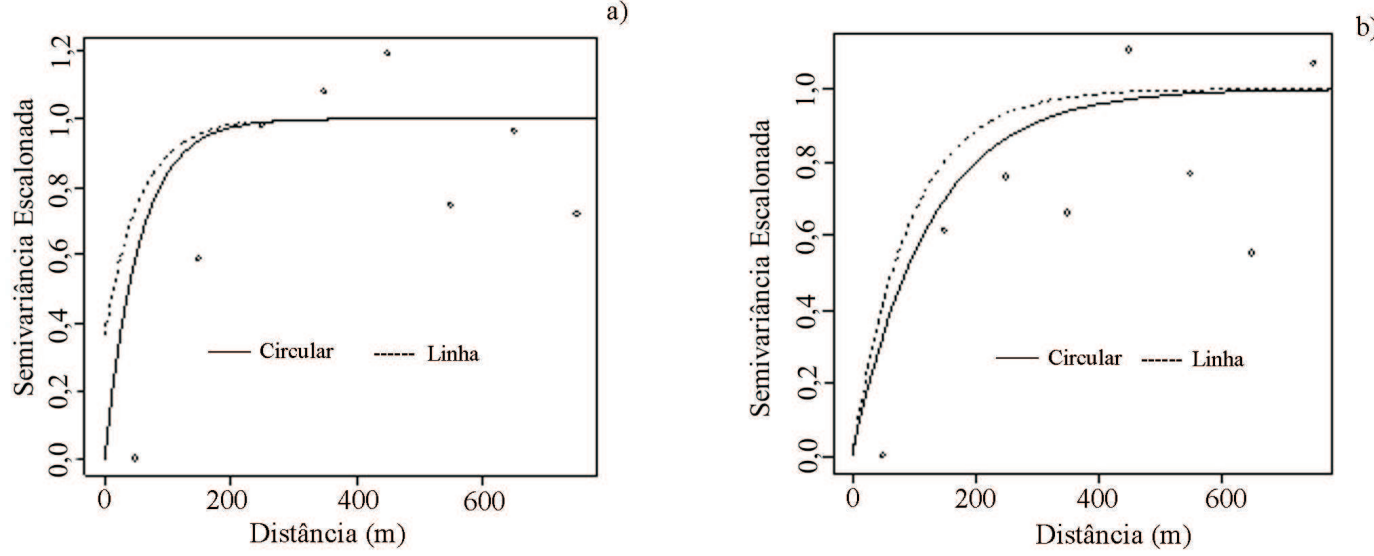

b)

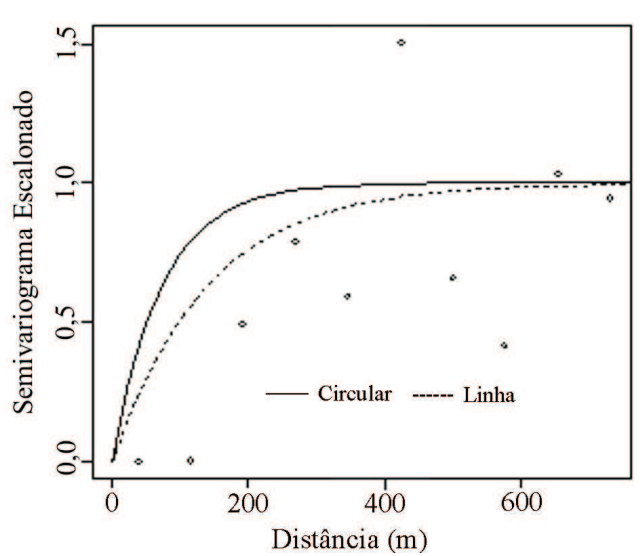

c)

Figura 7 - Semivariogramas escalonados comparando a forma das parcelas nas intensidades amostrais de 1:4 (a), 1:7 (b) e 1:10 (c).

Figure 7 - Scaled semivariograms comparing plot shapes at different sample intensities: 1:4 (a), 1:7 (b) and 1:10(c).

Os resultados evidenciaram a possibilidade de se utilizar o processo de krigagem para número de fustes e volume, como base para definição da amostragem. Assim, é importante comparar os mapas de krigagem nas diferentes situações, a fim de se estabelecer vínculo com a classificação de sítio e com a amostragem, propiciando melhor distribuição das parcelas e, consequentemente, melhor captação da variabilidade espacial dessa característica na floresta. Para isso, é necessário que haja estabilidade no porcentual de área em cada classe de produtividade e, por consequência, no porcentual amostrado em cada classe gerada pelo respectivo mapa de krigagem.

Na Figura 8 estão os mapas de krigagem de cada situação avaliada. Foi possível detectar que houve diferença espacial das classes de produtividade para as situações avaliadas. Porém, a tendência de maior ou menor produtividade ficou direcionada na mesma posição do mapa. Outra questão é que, além da diferenciação na espacialização das classes de produtividade, houve mudanças no porcentual de área de uma mesma classe entre os mapas (Tabela 2). Portanto, por meio dos mapas de krigagem verificou-se que houve diferenças entre os modelos espaciais, conforme a forma e intensidade amostrais, para a característica volume $\left(\mathrm{m}^{3} / \mathrm{ha}\right)$. Tal fato influenciou o modelo espacial e, consequentemente, o mapa de krigagem. Esse ponto é relevante quando se pensa em estratificação da floresta, a partir do mapa de krigagem. As classes de produtividade (estratos) podem apresentar variações em dimensão, mas a tendência foi semelhante em todas as situações. Portanto, são mapas úteis para alocação das parcelas no campo, buscando a representatividade espacial, que é benéfica para o resultado final do inventário florestal. 


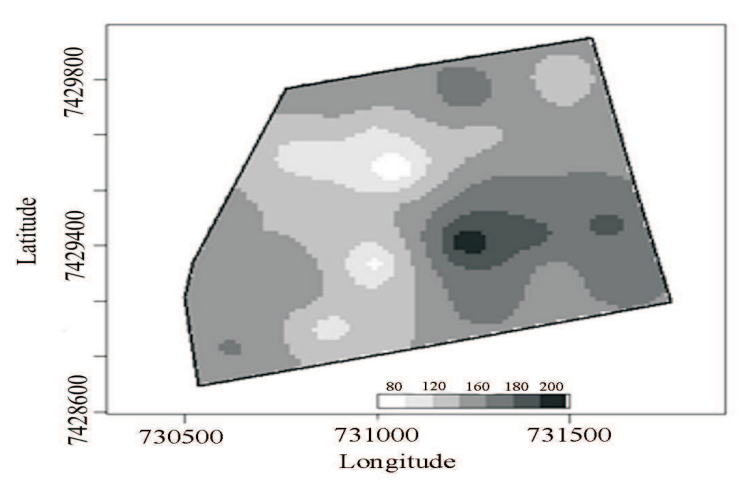

a)

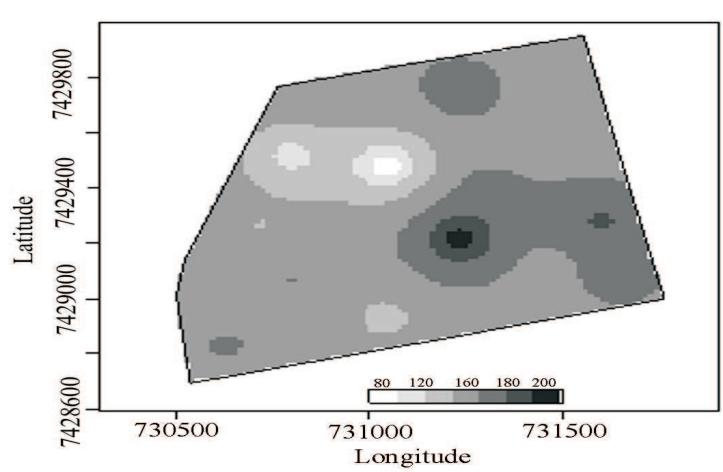

b

c)
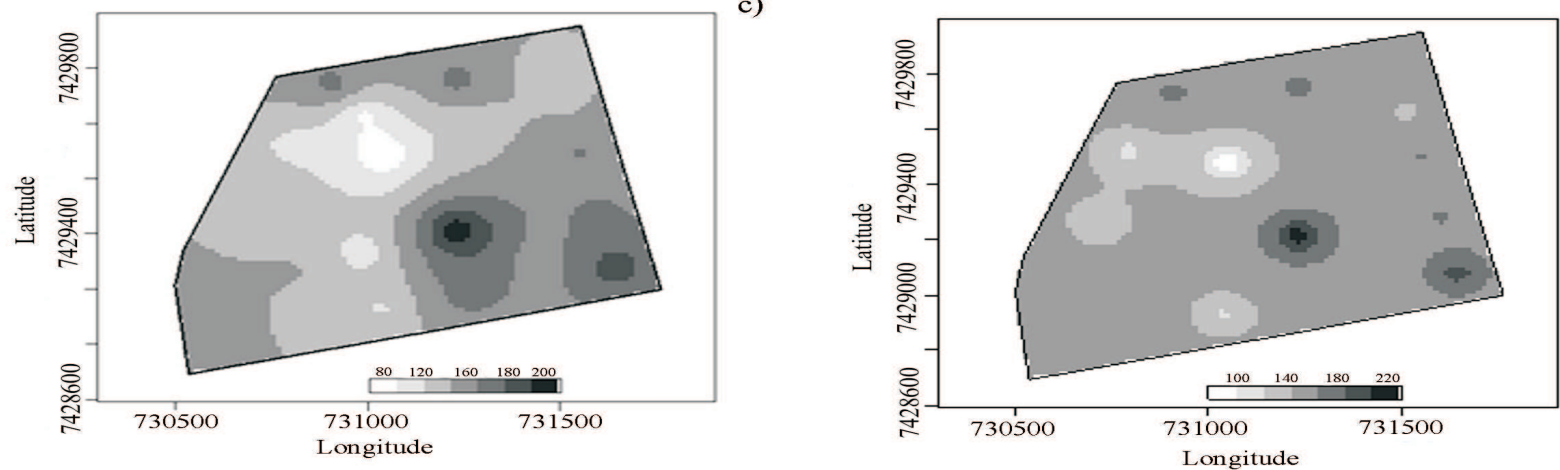

d)

Figura 8 - Mapas de krigagem gerados a partir das informações de diferentes formas de parcela e de diferentes intensidades amostrais: parcela circular 1:4 (a), parcela circular 1:7 (b), parcela em linha 1:4 (c) e parcela em linha 1:7 (d).

Figure 8-Kriging maps from different plot shapes and sample intensities: circular plots 1:4 (a), circular plots 1:7 (b), in line plots 1:4 (c), in line plots 1:7 (d).

Tabela 2 - Comparação entre as formas de parcelas e intensidades amostrais das diferentes classes de produtividades definidas pelo mapa de krigagem

Table 2 -Comparison between plot shapes and sample intensities for different productivity classes defined in the kriging maps

\begin{tabular}{|c|c|c|c|c|c|c|c|c|c|}
\hline \multirow[t]{2}{*}{ Centro Classe } & \multicolumn{3}{|c|}{ Circular } & \multicolumn{3}{|c|}{ Linha } & \multirow{2}{*}{$\frac{\text { Circular }}{1: 4}$} & \multirow{2}{*}{$\frac{\text { Linha }}{1: 4}$} & \multirow[t]{2}{*}{ Linha/Circ } \\
\hline & $1: 4$ & $1: 7$ & $1: 7 / 1: 4$ & $1: 4$ & $1: 7$ & $1: 7 / 1: 4$ & & & \\
\hline $\mathrm{m}^{3} / \mathrm{ha}$ & ha & ha & $\%$ & ha & ha & $\%$ & ha & ha & $\%$ \\
\hline 200 & 0,89 & 0,65 & $73 \%$ & 0,73 & 0,48 & $66 \%$ & 0,89 & 0,73 & $82 \%$ \\
\hline 180 & 4,36 & 2,19 & $50 \%$ & 2,75 & 1,19 & $43 \%$ & 4,36 & 2,75 & $63 \%$ \\
\hline 160 & 20,58 & 22,47 & $109 \%$ & 13,57 & 5,82 & $43 \%$ & 20,58 & 13,57 & $66 \%$ \\
\hline 140 & 43,75 & 66,29 & $152 \%$ & 38,39 & 82,01 & $214 \%$ & 43,75 & 38,39 & $88 \%$ \\
\hline 120 & 26,68 & 10,48 & $39 \%$ & 39,06 & 13,57 & $35 \%$ & 26,68 & 39,06 & $146 \%$ \\
\hline 100 & 7,63 & 2,28 & $30 \%$ & 8,57 & 1,37 & $16 \%$ & 7,63 & 8,57 & $112 \%$ \\
\hline 80 & 0,82 & 0,34 & $42 \%$ & 1,63 & 0,26 & $16 \%$ & 0,82 & 1,63 & $199 \%$ \\
\hline
\end{tabular}

\section{CONCLUSÕES}

As características número de fustes e volume de madeira apresentaram-se estruturadas espacialmente, portanto há modelo espacial que se ajusta a essa estrutura. O modelo exponencial foi o que se ajustou melhor aos semivariogramas experimentais das características nas diferentes formas de parcela e de intensidade amostral.
Para número de fustes, a continuidade espacial foi detectada em todas as intensidades amostrais e formas de parcelas avaliadas. Portanto, o uso da estatística espacial no processamento dessa variável aumentará a precisão das estimativas.

No caso de volume, a continuidade espacial não foi detectada na intensidade amostral 1:10. Nessa 
condição, o processamento do inventário florestal devese basear na estatística clássica. Quanto às demais intensidades avaliadas, deve-se utilizar a estrutura de continuidade espacial, a fim de obter estimativas consistentes da característica avaliada, além de propiciar mapas que auxiliam na alocação de parcelas no procedimento de amostragem.

\section{REFERÊNCIAS}

BIONDI, F.; MYERS, D. E.; AVERY, C. C. Geostatistically modeling stem size and increment in an old-growth forest. Canadian Journal of Forest Research, v.24, p.1354-1368, 1994.

BUSSAB, W. O.; MORETTIN, P. A. Estatística básica. 5.ed. São Paulo: Saraiva, 2002. 526p.

CAMBARDELLA, C. A. et al. Field scale variability of soil properties incentral lowa soils. Soil Science Society of America Journal, v.58, p.1501-1511, 1994.

CRESSIE, N. Statistics for spatial data. New York: Wiley, 1993. 900p.

COCHRAN, W. G. Sampling techniques. 3.ed. New York: Wiley, 1977. 555p.

DIGGLE, P. J.; RIBEIRO JÚNIOR, P. J. Model-based geostatistics. Londres: Springer, 2007. 230p.

ISAAKS, E. H.; SRIVASTAVA, R. M. An introduction to applied geostatistics. New York: Oxford University Press, 1989. 560p.

JOURnel, A. G.; HUIJBREGtS, C. J. Mining geostatistics. London: Academic Press, 1978. 600p.

LIMA, J. S. S. et al. Estudo da viabilidade de métodos geoestatísticos na mensuração da variabilidade espacial da dureza da madeira de paraju (Manilkara sp). Revista Árvore, v.30 n.4, p. 651-657, 2006.

MATÉRN, B. Spatial variation: stochastic models and their application to some problems in forest surveys and other sampling investigations. Stockholm: Skogsforsknings Institut, 1960. 144p. (Skogsforsknings Institut. Meddelanden fran Statens Skogsforsknings Institut, v.49, n.5).

R. Árvore, Viçosa-MG, v.33, n.1, p.185-194, 2009
MATHERON, G. Principles of geostatistics. Economic Geology, v.58, n.8, p.1246-1266, 1963.

Mc BRATNEY, A. G.; WEBSTER, A. G. Choosing functions for semi-variograms and fitting them to sampling estimates. Journal of Soil Science, v.37, n.3, p.617-639, 1986.

MELLO, J. M. et al. Estudo da dependência espacial de características dendrométricas para Eucalyptus grandis. CERNE, v.11, n.2, p.113-126, 2005.

MELLO, J. M. Geoestatística aplicada ao inventário florestal. 2004. 110f. Tese (Doutorado emRecursos Florestais) - Escola Superior de Agricultura "Luiz de Queiroz", Piracicaba, 2004.

NOVAES FILHO, J. P. et al. Distribuição espacial de carbono em solo sob floresta primária na Amazônia meridional. Revista Árvore, v.31, n.1, p.83-92, 2007.

OSBORNE, J. G. Sampling errors of systematic and random surveys of cover type areas. Journal of the Statisticacal Association, v.37, p.256-264, 1942.

RIBEIRO JÚNIOR, P. J. Métodos geoestatísticos no estudo da variabilidade espacial de parâmetros do polo. 1995. 99f. Dissertação (Mestrado emEstatística e Experimentação Agronômica) Escola Superior de Agricultura "Luiz de Queiroz", Piracicaba 1995.

RIBEIRO JÚNIOR, P. J.; DIGGLE, P. J. geoR: A package for geostatistical analysis. R-NEWS, v.1, n.2, p.15-18, 2001.

SCOLFORO, J. R. S.; MELLO, J. M. Inventário florestal. Lavras: UFLA/FAEPE, 2006. 561p.

THOMPSON, S. K. Sampling. New York: Wiley, 1992.343p.

VIEIRA, S. R. Geoestatística em estudos de variabilidade espacial do solo. In: NOVAIS, R.F.; ALVAREZ V., V.H. \& SCHAEFER, C.E.G.R., (Eds.) Tópicos em ciências do solo. Viçosa, MG, Sociedade Brasileira de Ciência do Solo, 2000.v.1, p.2-54. 